\title{
Targeted dual-color silica nanoparticles provide univocal identification of micrometastases in preclinical models of colorectal cancer
}

This article was published in the following Dove Press journal:

International Journal of Nanomedicine

5 September 2012

Number of times this article has been viewed

\author{
Marco Soster ${ }^{1, *}$ \\ Riccardo Juris ${ }^{2, *}$ \\ Sara Bonacchi ${ }^{2}$ \\ Damiano Genovese ${ }^{2}$ \\ Marco Montalti ${ }^{2}$ \\ Enrico Rampazzo ${ }^{2}$ \\ Nelsi Zaccheroni ${ }^{2}$ \\ Paolo Garagnani² \\ Federico Bussolino 3,4 \\ Luca Prodi ${ }^{2}$ \\ Serena Marchiò 1,4 \\ 'Institute for Cancer Research and \\ Treatment, Laboratory of Tumor \\ Microenvironment and University of \\ Torino, Department of Oncological \\ Sciences, Candiolo, ${ }^{2}$ University of \\ Bologna, Department of Chemistry \\ "G.Ciamician", Bologna, ${ }^{3}$ Institute \\ for Cancer Research and Treatment, \\ Laboratory of Vascular Oncology and \\ University of Torino, Department \\ of Oncological Sciences, Candiolo, \\ ${ }^{4}$ APAvadis Biotechnologies, Biolndustry \\ Park S Fumero, Colleretto Giacosa, \\ Italy \\ *These authors contributed equally \\ to the work
}

Correspondence: Serena Marchiò Institute for Cancer Research and Treatment, Laboratory of Tumor Microenvironment, and University of Torino, Department of Oncological

Sciences, sp $142 \mathrm{Km} 3.95$,

10060 Candiolo, Italy

Tel +390 II 9933507

Fax +3901I 9933524

Email serena.marchio@ircc.it
Background and methods: Despite the recent introduction of targeted bio-drugs, the scarcity of successful therapeutic options for advanced colorectal cancer remains a limiting factor in patient management. The efficacy of curative surgical interventions can only be extended through earlier detection of metastatic foci, which is dependent on both the sensitivity and specificity of the diagnostic tools.

Results: We propose a high-performance imaging platform based on silica-poly(ethylene glycol) nanoparticles doped with rhodamine B and cyanine 5. Simultaneous detection of these dyes is the basis for background subtraction and signal amplification, thus providing high-sensitivity imaging. The functionalization of poly(ethylene glycol) tails on the external face of the nanoparticles with metastasis-specific peptides guarantees their homing to and accumulation at target tissues, resulting in specific visualization, even of submillimetric metastases.

Conclusions: The results reported here demonstrate that our rationally designed modular nanosystems have the ability to produce a breakthrough in the detection of micrometastases for subsequent translation to clinics in the immediate future.

Keywords: colorectal cancer, imaging platform, luminescent targeting, micrometastasis, peptide targeting

\section{Introduction}

Cancer remains a leading cause of death in the Western world. There is still no effective cure for certain specific tumors and, in general, for advanced tumors, the scarcity of successful treatment options represents a serious limiting factor in patient management. Approximately $20 \%$ of colorectal cancer (CRC) patients present with a metastatic disease at diagnosis, and $30 \%-40 \%$ of patients with localized disease will ultimately develop metastases. Despite the introduction of novel therapeutic agents, combinations, and protocols, as the tumor stage increases, the 5-year overall survival rates decline substantially. ${ }^{1-3}$ The increased efficacy of curative surgical interventions can only be achieved through earlier detection of metastatic foci, dependent on both the sensitivity and specificity of the diagnostic tools. ${ }^{3,4}$ In order to overcome the limits of current approaches, two promising strategies can be adopted: improving diagnostic protocols to allow detection of tumors at earlier stages and/or with higher specificity and utilizing novel molecularly-targeted and personalized therapies to focus on tumor cells with increasing efficiency and selectivity. Recent developments in the design of nanosized materials have provided powerful tools for high-potential perspectives in both strategies. Modular nanometric systems have proved to be versatile platforms for combining imaging and drug delivery for applications in the oncological field. 5,6 
Among these, silica-based nanoparticles are now emerging as an attractive strategy for cancer theranostics, due to a favorable toxicological profile and biocompatibility in vivo coupled to an inherent versatility of manipulation in vitro. ${ }^{7,8}$

Here, we propose a rationally designed high-performance imaging platform based on fluorescent silica-poly(ethylene glycol) nanoparticles (SPNs) derivatized with two metastasisspecific peptides. Their efficient accumulation in target tissues results in specific visualization, even of submillimetric metastases.

\section{Materials and methods Chemicals and antibodies}

Pluronic $^{\circledR}$ F127 (PF-127), tetraethyl orthosilicate (TEOS, 99.99\%), chlorotrimethylsilane (TMSCl, $\geq 98 \%$ ), $\mathrm{HCl}$ $(\geq 37 \%), \mathrm{AlMe}_{3}$ (toluene solution $2.0 \mathrm{M}$ ), Rhod base $(>98 \%)$, reagent grade dimethylformamide and diethylether $\left(\mathrm{Et}_{2} \mathrm{O}\right)$ were from Sigma-Aldrich (St Louis, MO). Piperazine ( $\geq 98.0 \%$ ), triethylamine ( $\geq 99.5 \%)$, 3-(triethoxysilyl) propylisocyanate $(\geq 95 \%)$, reagent grade dichloromethane, isopropyl alcohol, and $\mathrm{NaCl}$ were from Fluka (division of Sigma). Cy5 was from Cyanagen (Bologna, Italy). Peptides were from New England Peptides (Gardner, MA). Rat anti-CD31 antibody was from BD Biosciences (Franklin Lakes, NJ), secondary Alexa Fluor ${ }^{\circledR} 647 / 488$ antibodies were from Invitrogen (Carlsbad, CA), DyLight ${ }^{\mathrm{TM}} 405$ was from BioLegend (Uithoorn, The Netherlands).

\section{Synthesis of rhodamine derivatives}

The experimental procedure followed for the synthesis of rhodamine (Rhod) tertiary amide derivative has been described by Nguyen et al. ${ }^{9}$ Rhod tertiary amide $(0.91 \mathrm{mmol})$ and triethylamine $(14.5 \mathrm{mmol})$ were dissolved under $\mathrm{N}_{2}$ in anhydrous dimethylformamide (7.5 mL). 3-(Triethoxysilyl) propyl isocyanate $(27.4 \mathrm{mmol})$ and $\mathrm{CH}_{2} \mathrm{Cl}_{2}(10 \mathrm{~mL})$ were added drop-wise at room temperature (RT) while stirring. After $12 \mathrm{~h}$, the reaction mixture was dried under reduced pressure, and the deriving solid was solubilized in a minimal amount of ethanol and precipitated in $\mathrm{Et}_{2} \mathrm{O}(300 \mathrm{~mL})$. The resulting mixture was cooled under gentle stirring and the product was recovered by filtration. This dark red-purple solid was rinsed with $\mathrm{Et}_{2} \mathrm{O}$, re-crystallized with ethanol/ $\mathrm{Et}_{2} \mathrm{O}$, and recovered by filtration.

\section{Synthesis of the dicarboxylic acid derivative of Pluronic ${ }^{\circledR} \mathrm{FI} 27$ (PF- I27)}

PF-127 (1.0 mmol) and $\mathrm{NaIO}_{4}(8.2 \mathrm{mmol})$ were placed in a $250-\mathrm{mL}$ round-bottom flask. Water $(60 \mathrm{~mL})$, acetonitrile $(24 \mathrm{~mL})$, and carbon tetrachloride $(21 \mathrm{~mL})$ were added to this mixture; $\mathrm{RuCl}_{3}(0.044 \mathrm{mmol})$ was added to the resulting biphasic solution and the reaction mixture was stirred overnight at RT, followed by repeated extractions with $\mathrm{CH}_{2} \mathrm{Cl}_{2}$. The reunited organic phases were dried over $\mathrm{Na}_{2} \mathrm{SO}_{4}$, filtered, and evaporated.

\section{Synthesis of peptide-targeted SPNs}

SPNs were synthesized by adapting previously reported procedures. ${ }^{10}$ Briefly, PF-127 (14.40 mmol), carboxylic acid-derivatized PF-127 (1.60 mmol), and dyes $(3.20 \mu \mathrm{mol})$ were solubilized with dichloromethane $(2 \mathrm{~mL})$ in a $20-\mathrm{mL}$ glass scintillation vial. Solvent was evaporated under $\mathrm{N}_{2}$ and successively under vacuum at RT. $\mathrm{NaCl}$ (3.13 mmol) was added to the solid residue and the mixture was solubilized at RT while stirring with acetic acid (1 M, 3,130 $\mu \mathrm{L})$. tetraethylorthosilicate $(1.60 \mathrm{mmol})$ was added to the resulting solution, followed by tetramethylsilylchloride $(0.16 \mathrm{mmol})$ after $3 \mathrm{~h}$. The mixture was stirred for $48 \mathrm{~h}$ at RT before dialysis/ultrafiltration treatments. Dialysis was carried out in water in regenerated cellulose dialysis tubing (Sigma, $12 \mathrm{kDa}$ cut-off) at RT under gentle stirring. Ultrafiltration was performed in a $75-\mathrm{mL}$ stainless steel-glass cell with $47 \mu \mathrm{m}$ filters (EMD Millipore, Billerica, MA). To obtain peptidetargeted SPNs, MES buffer pH $6.0(0.2 \mathrm{M}, 40 \mu \mathrm{L})$, 1-ethyl3-(3-dimethylaminopropyl)carbodiimide hydrochloride (1.2 $\mu \mathrm{mol}$, corresponding to 20 equivalents of the carboxylic acid moieties) and N-hydroxysuccinimide (1.2 $\mu \mathrm{mol})$ were mixed with the SPN solution $(250 \mu \mathrm{L})$, which was stirred for $3 \mathrm{~h}$ at RT. Next, 10× Dulbecco's phosphate-buffered saline (DPBS, $240 \mu \mathrm{L}$ ) was added and the $\mathrm{pH}$ was adjusted to 7.2. Finally, CGIYRLRS or CGVYSLRS synthetic peptide ( $0.61 \mu \mathrm{mol}, 10$ equivalents) was added and stirred overnight at RT. Purification was performed by dialysis.

\section{Dynamic light scattering and transmission electron microscopy}

The SPN hydrodynamic diameter distribution was determined using different instruments depending on the absorption/ emission properties of the dyes. (Rhod)-SPNs were measured using a Malvern Nano ZS instrument (Malvern Instruments Ltd, Malvern, UK) equipped with a $633 \mathrm{~nm}$ laser diode. (Cy5)-SPNs were measured using a NICOMP Model 370 Submicron Particle Sizer (Nicomp International, Orlando, FL) equipped with a $488 \mathrm{~nm}$ Argon laser. The hydrodynamic diameter distribution is indicated by the polydispersity index (PdI); in the case of a mono-modal distribution calculated using cumulative analysis, $\mathrm{PdI}=\left(\sigma / \mathrm{Z}_{\mathrm{avg}}\right),{ }^{2}$ where $\sigma$ is the width of the distribution and $Z_{\text {avg }}$ is the average diameter of the particles. 
For transmission electron microscopy (TEM) analyses, a holey carbon foil supported on copper microgrids was dried under vacuum after depositing a drop of SPN solution diluted 1:50 in water. A Philips CM 100 TE microscope (Philips Research, Eindhoven, The Netherlands) operating at $80 \mathrm{kV}$ was used, and SPN size distribution was obtained by analyzing images with a block of several hundreds of nanoparticles.

\section{Photophysical measurements}

UV-VIS absorption spectra were recorded at RT using a Perkin-Elmer Lambda 45 spectrophotometer. Fluorescence spectra were recorded with an Edinburgh FLS920 instrument equipped with a Hamamatsu R928P photomultiplier. The same instrument, connected to a PCS900 PC card, was used for time-correlated single photon counting (TCSPC) analyses. Fluorescence quantum yields were determined using solutions of rhodamine $6 \mathrm{G}$ as a reference, and intensities were corrected for inner filter effects according to standard methods. ${ }^{10}$

\section{Cell cultures and human samples}

A highly metastatic variant of HCT-116 cells (ATCC CCL247), HCT-116 m, was provided by Dr Alberto Bardelli (IRCC, Candiolo, Italy). Cells were maintained in Iscove's modified Dulbecco's medium (IMDM) supplemented with $10 \%$ fetal calf serum (FCS). Fresh samples of grossly normal liver and colon, of primary CRC, and of hepatic metastasis secondary to CRC were collected by the Unit of Surgical Oncology at the IRCC. Collection and manipulation of human samples were approved by the Institute's Ethical Committee, and written informed consent was obtained from all patients in accordance with the Declaration of Helsinki.

\section{SPN overlay assays, histochemistry and $3 \mathrm{D}$ tissue reconstructions}

Optimal cutting temperature (OCT)-frozen human tissue specimens were cut into $10-\mu \mathrm{m}$ sections. For overlay experiments, SPNs $\left(10^{11} / \mu \mathrm{L}\right)$ were incubated with the tissue sections for $4 \mathrm{~h}$ at RT, followed by three washes in Tris-buffered saline containing $0.1 \%$ Tween-20 (TBS-T). Specific tissue binding, evaluated as emitted fluorescent signal, was detected using a DMIRE2 confocal microscope (Leica Microsystems, Milan, Italy). For prompt visualization of quantified signals, images were converted into a false color LUT Fire scale. Co-localized pixels were identified using ImageJ software (National Institute of Health, Bethesda, MA). To evaluate the homing of metastasis-targeted nanoparticles, SPN images were superimposed to blood vessel staining performed with a CD31-specific antibody, revealed by either Alexa Fluor ${ }^{\circledR} 647$ [overlay with (Rhod)SPNs], Alexa Fluor ${ }^{\circledR} 488$ [overlay with (CY5)-SPNs], or DyLight $^{\mathrm{TM}} 405$ [overlay with (Rhod+CY5)-SPNs] secondary antibodies. To reconstruct the 3D models of nanoparticles and tumor vessels, 50 to 80 confocal image series were composed using IMARIS v. 6.3.1 (Bitplane Scientific Software, Zurich, Switzerland).

\section{In vivo experiments}

Six-week-old female nonobese diabetic/severe combined immunodeficient (NOD/SCID) mice were purchased from Charles River (Lecco, Italy). Experiments involving animals were reviewed and approved by the Institute's ethical committee, and by the Italian Ministry of Health. Eight animals per group were subjected to intraperitoneal anesthesia with a mixture of xylazine $\left(\right.$ Xilor $^{\circledR}$, BIO98, Milan, Italy; $0.75 \mathrm{mg} / \mathrm{mL})$ and tiletamine-zolazepam (Zoletil ${ }^{\circledR}$, Virbac, Milan, Italy; $1 \mathrm{mg} / \mathrm{mL}$ each), in physiological solution. Once the mice were asleep, a midline incision was performed, the spleen was exposed, and HCT-116 m cells were injected $\left(2 \times 10^{6}\right.$ in $50 \mu \mathrm{L}$ of IMDM medium $)$. The wound was closed by a double suture and each animal was given caprofen (Rymadil $^{\circledR}$, Pfizer, Milan, Italy; $0.1 \mathrm{mg}$ ) in physiological solution for postoperative pain relief and rehydration. Mice were strictly monitored until completely awake, and oral ampicillin was administered for 5 days following the surgery. Between days 21-28, mice were injected intravenously with SPNs $\left(5 \times 10^{12}\right.$ in $200 \mu \mathrm{L}$ of saline solution). To evaluate nanoparticle homing, mice were euthanized after $16 \mathrm{~h}$, and organs were photographed using a MZ16FA fluorescence stereomicroscope equipped with a DFC420C (Rhod) and a DFC350FX (Cy5) digital camera (Leica).

\section{Results and discussion Dual-color SPNs: preparation and characterization}

We designed and tested metastasis-targeted nanoparticles based on silica-condensed, alkoxysilane-derivatized fluorescent dyes, Rhod and/or Cy5, within a micelle of the tri-block copolymer Pluronic ${ }^{\circledR}$ F127 (PF-127), resulting in a poly(ethylene glycol) (PEG) shell embedding a dyedoped silica core (Figure 1A). The absorption and emission properties of Rhod and Cy5 in single-color SPNs are the same as the free molecules (Table S1), with the exception of a slight red-shift of $\lambda_{\max }$, indicating absence of interactions between the encapsulated dyes at the ground state for the 

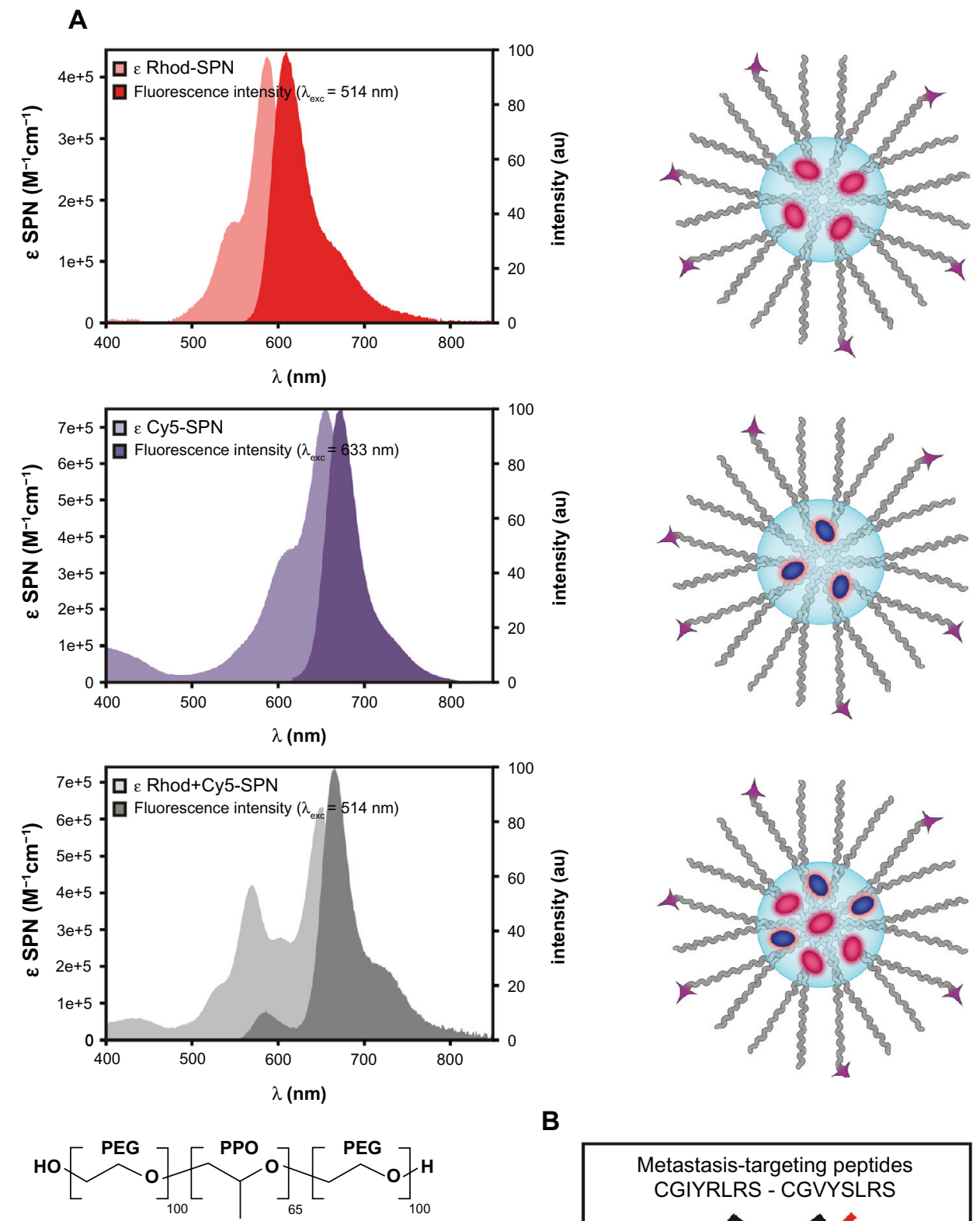

B
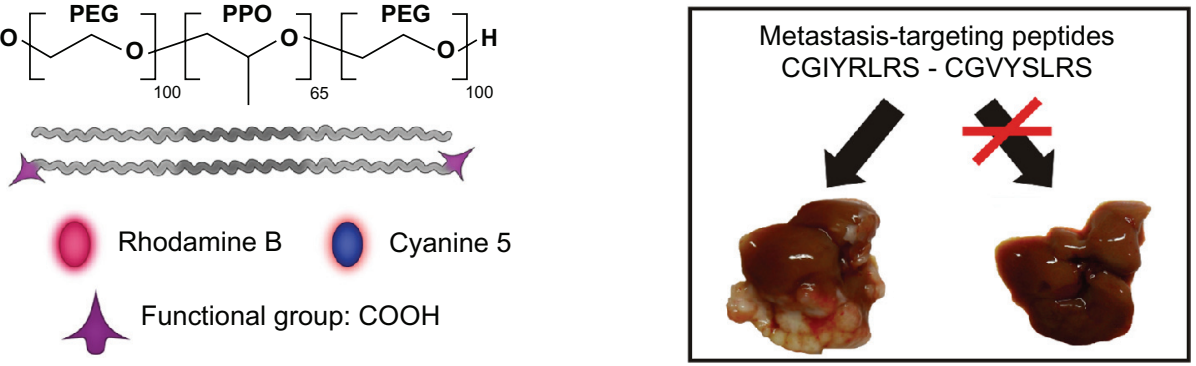

Figure I Single- and dual-color targeted SPNs. (A) Schematic representation of SPNs characterized by a COOH-derivatized PF-I 27 shell embedded in a silica/dye core. For each sample of single- and dual-color SPNs, the corresponding absorption and emission spectra in water solution are reported. (B) CGIYRLRS and CGVYSLRS peptides were selected by phage display biopanning of random libraries on matched hepatic metastasis/normal liver tissue pairs from patients with advanced CRC, and were demonstrated to be specific ligands for the metastatic tissue.

Abbreviations: CRC, colorectal cancer; PF-127, Pluronic ${ }^{\circledR}$ FI27; SPN, silica-poly(ethylene glycol) nanoparticles.

chosen doping degrees. Remarkably, both dyes showed increased fluorescence quantum yields (Table S1) as a consequence of entrapment in the rigid silica matrix; this prevents geometrical rearrangements of the excited state (that can lead to nonradiative processes) and segregates the dyes from possible quenchers, including molecular dioxygen. ${ }^{5}$ Dual-color SPNs exhibit different fluorescence properties (Table S1) due to the energy transfer (90\% efficiency) from Rhod to Cy5. This allows a large separation between emission and excitation wavelengths; as a consequence, when 
these SPNs are excited in the Rhod absorption band, Rhod fluorescence decreases to $10 \%$, with a concomitant increase in Cy5 emission. ${ }^{11}$ Residual Rhod intensity is however sufficiently high to be exploited for imaging purposes, allowing a concomitant detection of the two fluorescence signals and background signal minimization.

\section{Dual-color SPNs: functionalization and coupling with metastasis-targeting moieties}

The external PEG chains of PF-127 provide a standard of stealth polymer $^{12}$ for stable dispersion in physiological conditions and for prevention of uptake by the mononuclear phagocyte system, all features that render our SPNs an ideal choice for in vivo applications. We derivatized the PEG tails with carbonyl groups to enable covalent attachment of targeting ligands, eg, antibodies, proteins, or short peptides. The resulting SPNs have a core diameter of $11 \pm 3 \mathrm{~nm}$, as measured by TEM (Figure $\mathrm{S} 1 \mathrm{~A}$ ), and a hydrodynamic diameter of $23 \mathrm{~nm}$ with a very low PdI ( $<0.1$, Figure S1B). These features of the SPNs are reproducible among different preparations, independent from the embedded dye, and they are very stable (no aggregation, degradation or dye leakage even after several months of storage, data not shown). We surface-functionalized our SPNs with two peptides, CGIYRLRS and CGVYSLRS, that have been isolated in our laboratory as specific ligands for the microenvironment of human metastatic CRC (Figure 1B). These peptide sequences were derived by comparative phage display bio-panning on fresh surgical samples of hepatic metastases versus matched normal liver biopsies and proved to be selective for the stromal and the epithelial components of the metastasis. ${ }^{13}$

\section{Ex vivo validation of SPN targeting and imaging properties}

We first investigated the binding properties of the deriving metastasis-targeted, dual-color SPNs ex vivo on human specimens of surgically removed hepatic metastasis (Figure 2; a parallel analysis of single-color SPNs is reported in Figure S2). For this purpose, 10- $\mu \mathrm{m}$ sections of OCT-frozen human tissues were incubated with control (untargeted) and peptide-targeted (Rhod+Cy5)-SPNs. The localization of specific fluorescent signals was evaluated using confocal microscopy (Figure 2A-D), and quantified by pixel intensity count (Figure 2E). In these assays, both CGIYRLRS- and CGVYSLRS-(Rhod+Cy5)-SPNs showed binding selectivity for hepatic metastasis (Figure 2B) compared to grossly normal liver (Figure 2A) and colon (Figure 2C) or primary CRC (Figure 2D). The concomitant presence of dyes with separate excitation/emission spectra allows nearly complete elimination of background fluorescence, through the identification of univocal signals by fluorescence overlay. In these ex vivo binding assays, imaging of co-localized Rhod/Cy5 pixels revealed sharp SPN staining of epithelial cell membranes and stroma that was specifically associated with hepatic metastasis tissues (Figure 2B, overlay).

\section{Set-up of animal models of human metastatic CRC for targeted imaging}

We then investigated the capability of our peptide-targeted nanoparticles to specifically target hepatic metastasis in vivo in a clinically relevant mouse model of human metastatic CRC. For this purpose, NOD/SCID mice were injected intrasplenically with the highly metastatic CRC cell line HCT-116 m. In this well-established model, 21-28 days after cell injection animals develop a pseudo-orthotopic primary tumor in the spleen and several secondary tumors into the liver ${ }^{14}$ Within this time frame, we injected either control or CGIYRLRStargeted single- or dual-color SPNs into the caudal vein and let them circulate for increasing periods of time starting from $1 \mathrm{~h}$. This choice was based on our recently published data, showing a peak in the fluorescence emission in different organs, including the liver, during the first hour after untargeted SPN injection and a subsequent decrease with time. ${ }^{5}$ Consistently, we observed a good reduction of nonspecific fluorescence (ie, the signals of untargeted SPNs in target tissues, and/or of targeted SPNs in nontarget tissues) after $6 \mathrm{~h}$, maintaining this optimal signal-to-background ratio even after $16-24 \mathrm{~h}$. A very similar outcome was obtained with CGVYSLRS-SPNs (data not shown). Nontargeted contrast agents have the disadvantage of being quickly removed from the bloodstream, thus requiring analysis to be performed shortly after injection (usually in the period of just minutes). Often, this does not enable precise quantifications or allow for repeated analyses. Conversely, we recently demonstrated that SPNs (including untargeted) persist in the bloodstream for up to $3 \mathrm{~h}^{5}{ }^{5}$ The results of the experimental set-up described above suggest that clearance of untargeted nanoparticles is paralleled by specific accumulation of targeted SPNs that remains constant for longer periods (up to $24 \mathrm{~h}$ ). The possibility of exploiting such a wide time frame for diagnostic imaging of hepatic micrometastases further supports translation into clinical applications.

\section{In vivo imaging of hepatic micrometastases by targeted SPNs}

We evaluated the in vivo location of single- and dual-color SPNs; in both cases, we observed specific accumulation 


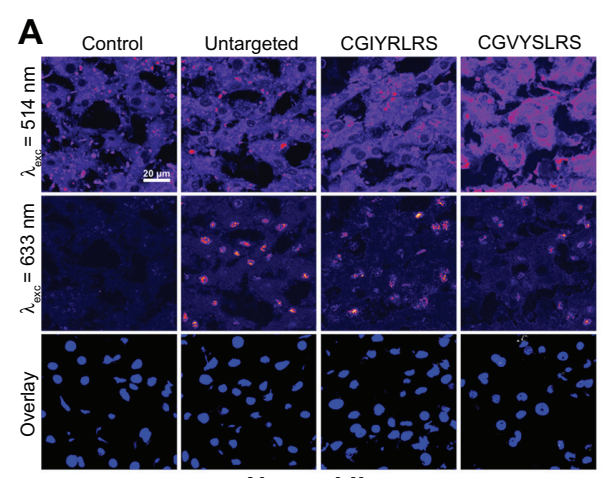

Normal liver

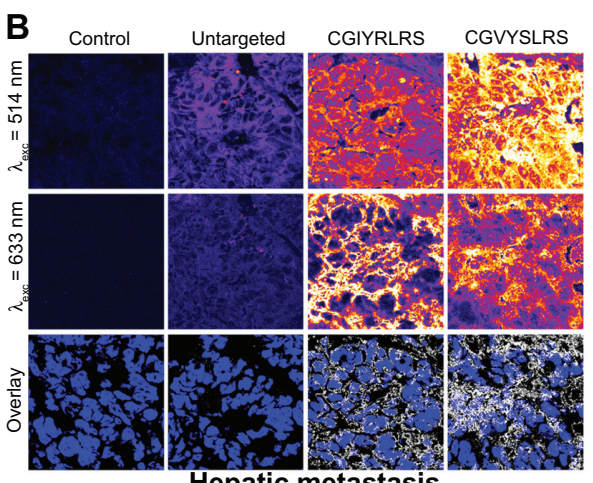

Hepatic metastasis

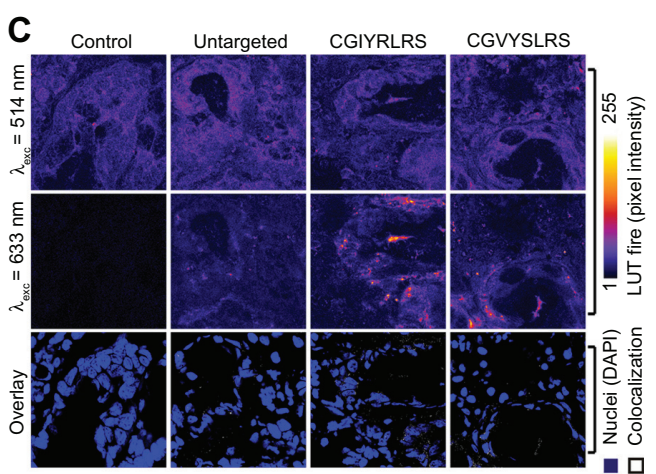

Normal colon

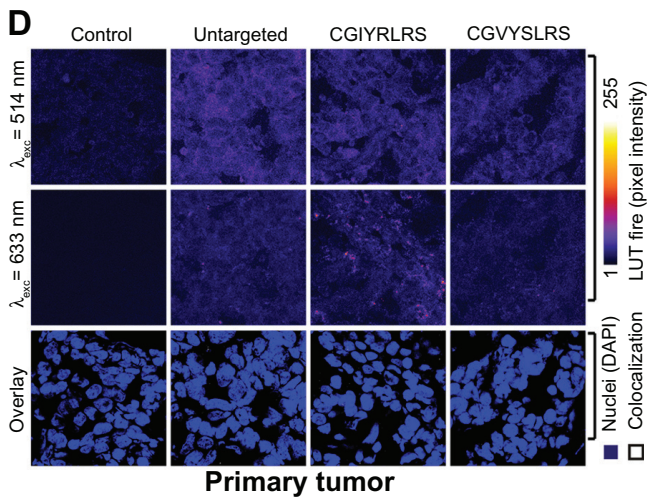

$\mathbf{E}$

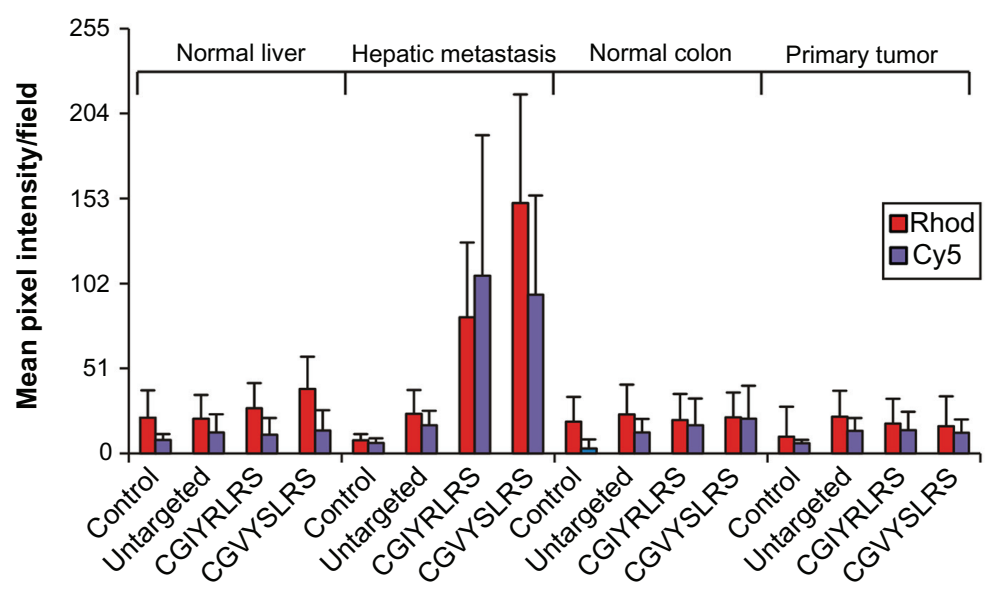

Figure 2 Targeted SPNs recognize human hepatic metastases ex vivo. Frozen 10- $\mu \mathrm{m}$ sections of (A) matched (grossly) normal liver, (B) hepatic metastasis, (C) (grossly) normal colon, and (D) primary tumor were fixed in $4 \%$ formaldehyde, before incubation with either untargeted, CGIYRLRS-, or CGVYSLRS-(Rhod+Cy5)-SPNs for $4 \mathrm{~h}$ at RT. After washing, the SPN-emitted fluorescence was analyzed using confocal microscopy keeping all parameters constant, and the output was converted into a false color LUT Fire for clear visualization. Colocalized pixels were identified using ImageJ software. Experiments were performed, giving similar results, on specimens from ten patients with metastatic CRC; exemplary images from tissues of patient \#P85 are shown. (E) Quantification of SPN binding is expressed as the intensity of emitted pixel following excitation at $514 \mathrm{~nm}$ (Rhod) and $633 \mathrm{~nm}(\mathrm{Cy} 5)$, and calculated as a mean value of five images for each tissue. Cell nuclei were stained with 4',6-diamidino-2-phenylindole and are visualized in blue. All tissue slices were imaged in the same way.

Abbreviations: CRC, colorectal cancer; Rhod, rhodamine; RT, room temperature; SPN, silica-poly(ethylene glycol) nanoparticles.

of targeted nanoparticles in hepatic metastases compared to normal surrounding tissues (Figure 3D-F) or to untargeted nanoparticles (Figure 3A-C). CGIYRLRS(Rhod)-SPNs were exclusively visible in blood vessels of larger masses (Figure 3D), although they also accumulated in both microvessels and in cancer cells in masses with a volume $<1-2 \mathrm{~mm}^{3}$ (Figure 3D-4). The signal-to-background ratio obtained with CGIYRLRS-(Rhod)-SPNs was generally low, despite being extremely specific, probably due to scarce light penetration and/or relatively high tissue autofluorescence at the analyzed wavelengths. Conversely, the signal obtained with CGIYRLRS-(Cy5)-SPNs was both specific and highly intense, allowing the visualization of microvessels as well as submillimetric metastatic masses (Figure 3E). 

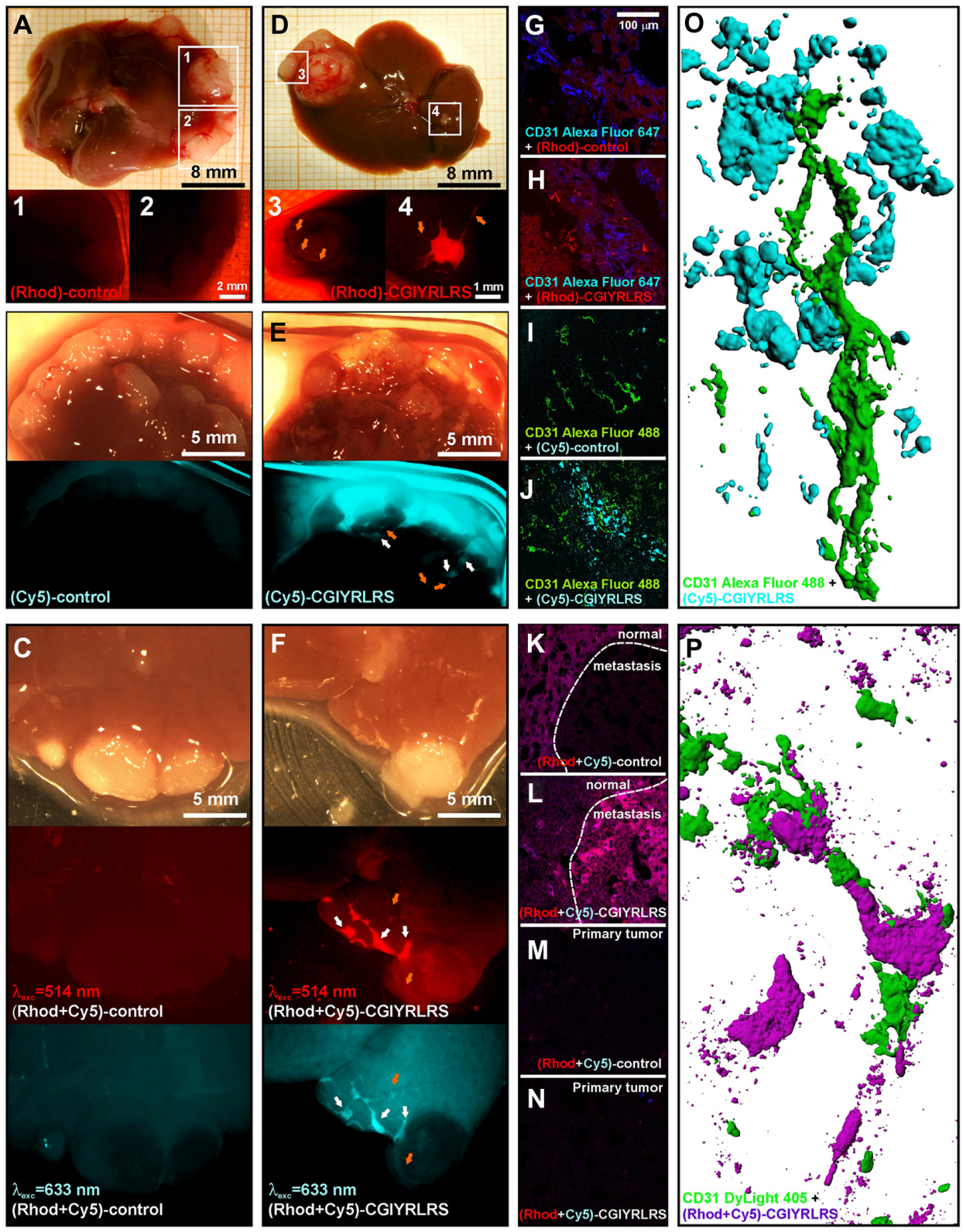

Figure 3 Targeted SPNs home to hepatic metastases in vivo. NOD/SCID mice bearing a primary spleen tumor and multiple liver metastases were injected intravenously with single- [control (A, Rhod; B, Cy5) or CGIYRLRS- (D, Rhod; E, Cy5)] or dual-color [control (C) or CGIYRLRS (F)] SPNs. After 16 h, mice were euthanized and the explanted organs were photographed in toto using high-resolution CCD cameras connected to a fluorescence stereomicroscope. In d, e, and f, orange arrows indicate blood vessels crossing the hepatic metastasis; in e and $\mathrm{f}$, white arrows indicate submillimetric metastatic foci. Samples of the same tissues were OCT-frozen, cut into I0- $\mu \mathrm{m}$ slices, and evaluated at a micrometric scale by confocal analysis of the single- [control (G, Rhod; I, Cy5), CGIYRLRS (H, Rhod; J, Cy5)] or dual-color [control (K) or CGIYRLRS (L)] SPN-emitted fluorescence. To visualize blood vessels, staining for CD3I was superimposed on the SPNs signal, and was visualized using secondary antibodies Alexa Fluor ${ }^{\circledR} 647$ ( $\mathbf{G}$ and $\mathbf{H}$ ) and Alexa Fluor ${ }^{\circledR} 488$ (I and J), for overlay with (Rhod)-SPNs and (Cy5)-SPNs, respectively. In the case of dual-color nanoparticles, a further control is visualized, ie, samples of primary tumors from mice injected with either control (M) or CGIYRLRS (N) SPNs. Microphotographs $\mathbf{G}-\mathbf{J}$ and $\mathbf{M}-\mathbf{N}$ refer to a single tissue type (metastasis and primary tumor, respectively); microphotographs $\mathrm{k}$ and I depict the interface between normal liver and hepatic metastasis; in this second case, a dotted line has been added to separate the two tissues, as indicated on the photo. Fifty to 80 series of images superimposing CD3I were stained with either (Cy5)-SPNs ( $\mathbf{O}$, particular of the field in J) or (Rhod+Cy5)-SPNs (P, particular of the field in L, in which CD3I was visualized using the secondary antibody DyLightTM405). SPNs were composed to reconstruct 3D models using IMARIS

Abbreviations: NOD/SCID, nonobese diabetic/severe combined immunodeficient; OCT, optimal cutting temperature; SPN, silica-poly(ethylene glycol) nanoparticles.

Except for the strongly improved signaling efficiency, accumulation of the dual-color targeted SPNs was comparable to that of single-color nanoparticles (Figure 3F).

Confocal microscopy analysis of explanted organs confirmed the specific presence of the SPNs at hepatic metastases, with an accumulation of both CGIYRLRS(Rhod)-SPNs (Figure 3H) and CGIYRLRS-(Cy5)-SPNs (Figure 3J) in close proximity to blood vessels. In contrast, no specific emission was detected in metastatic tissues of mice injected with control nanoparticles (Figure 3G and I). In 
the case of the dual-color SPNs, the possibility of overlaying fluorescent signals in the channels of the two dyes allowed precise identification of the nanoparticles compared to tissue background, leading to univocal imaging of targeted SPNs in metastatic tissues compared to normal liver (Figure 31), primary splenic tumor (Figure $3 \mathrm{~N}$ ), or control SPNs (Figure $3 \mathrm{~K}$ and $\mathrm{M}$ ). We used IMARIS software to compose several z-stacks obtained by confocal microscopy and to reconstruct a 3D model of selected portions of hepatic metastases from animals injected with CGIYRLRS-(Cy5)-SPNs (Figure 3O) or CGIYRLRS-(Rhod+Cy5)-SPNs (Figure 3P). These images clearly show that targeted SPNs extravasate from the fenestrate tumor blood vessels and cluster in both endothelial/perivascular and nearby tumor epithelial cells and stroma, suggesting that specific molecular targets are easily accessible to circulating nano-objects in pathological microenvironments.

\section{Conclusions}

Different research groups have recently described the use of fluorescence-based tracers during surgery to improve identification of pathologic tissues; for example, tracers are used in prostate and gastric cancers to identify sentinel lymph nodes, ${ }^{15,16}$ as well as in bladder ${ }^{17}$ and ovarian cancers. ${ }^{18}$ Preliminary data have also been reported in the diagnosis of externally accessible human cancers, such as the detection of superficial malignancies (nonmelanoma skin tumors). ${ }^{19}$ In addition, methods have been developed for endoscopic fluorescence-based imaging systems for CRC. ${ }^{20}$ However, most were obtained with untargeted fluorescent compounds, in which case the recognition of a pathologic site is nonspecific, only relying on the different physical features of tumoral versus healthy tissues.

Results reported here demonstrate that coupling specific molecular targeting, ie, a ligand (peptide on the SPN)/receptor (biomarker in the diseased site) interaction, to the distinctive photophysical features of our SPNs leads to a marked improvement in both specificity and sensitivity of current diagnostic imaging, allowing visualization of submillimetric metastases. The outer Food and Drug Administrationapproved PEG shell, yielding stable probes, unaffected by the mononuclear phagocyte system, infers further valuable characteristics to our system. The experimental evidence discussed here demonstrates that our rationally designed modular nanostructures have the required characteristics for translation to clinical practice in the immediate future. Notably, using the modular approach that we have proposed here, a plethora of combinations can be engineered to achieve cancer cell-targeted delivery of a number of therapeutic and diagnostic compounds.

\section{Acknowledgments}

This work was supported by the Italian Federation for Cancer Research (FIRC), Italian Association for Cancer Research - My First AIRC Grant (AIRC-MFAG), Banca d'Alba, Piedmont Region (Finalized Health Research Under 40), and Piedmont Foundation for Cancer Research (FPRC) Intramural Grant $5 \times 10002008$ (to SM); AIRC, European Union (LSHM-CT-2003-503254), Piedmont Region (Finalized Health Research; Industrial Research and Precompetitive Development: grants PRESTO and SPLASERBA; Technological Platforms for Biotechnology, grant DRUIDI; Converging Technologies, grant PHOENICS; Industrial Research, grant BANP), Cassa di Risparmio di Torino (CRT) Foundation and Italian Ministry of Health (Oncological Research Program; Finalized Research and Founding for Investments in Basic Research, FIRB, grant RBAP11BYNP-Newton) (to FB); Italian Ministry of University and Research (MIUR) Research project of National Interest (PRIN, grant 2009Z9 ASCA), EU Seventh Framework Programme European Research Area Network (ERA-Net) NanoSci-E+ (grant INOFEO), and European Research Council (ERC, starting grant MOSAIC) (to LP).

\section{Disclosure}

The authors report no conflicts of interest in this work.

\section{References}

1. O'Connell JB, Maggard MA, Ko CY. Colon cancer survival rates with the new American Joint Committee on Cancer sixth edition staging. J Natl Cancer Inst. 2004;96(19):1420-1425.

2. Wolpin BM, Mayer RJ. Systemic treatment of colorectal cancer. Gastroenterology. 2008;134(5):1296-1310.

3. Tomlinson JS, Jarnagin WR, DeMatteo RP, et al. Actual 10-year survival after resection of colorectal liver metastases defines cure. J Clin Oncol. 2007;25(29):4575-4580.

4. Gallagher DJ, Kemeny N. Metastatic colorectal cancer: from improved survival to potential cure. Oncology. 2010;78(3-4):237-248.

5. Rampazzo E, Boschi F, Bonacchi S, et al. Multicolor core/shell silica nanoparticles for in vivo and ex vivo imaging. Nanoscale. 2012;4(3): 824-830.

6. Majumdar D, Peng XH, Shin DM. The medicinal chemistry of theragnostics, multimodality imaging and applications of nanotechnology in cancer. Curr Top Med Chem. 2010;10(12):1211-1226.

7. Bonacchi S, Genovese D, Juris R, et al. Luminescent silica nanoparticles: extending the frontiers of brightness. Angew Chem Int Ed Engl. 2011;50(18):4056-4066.

8. Bonacchi S, Genovese D, Juris R, et al. Luminescent chemosensors based on silica nanoparticles. Top Curr Chem. 2011;300:93-138.

9. Nguyen T, Francis MB. Practical synthetic route to functionalized rhodamine dyes. Org Lett. 2003;5(18):3245-3248.

10. Montalti M, Credi A, Prodi L, Gandolfi MT. Handbook of Photochemistry, 3rd ed. Boca Raton, FL: CRC Press; 2006. 
11. Rampazzo E, Bonacchi S, Juris R, et al. Energy transfer from silica core-surfactant shell nanoparticles to hosted molecular fluorophores. J Phys Chem B. 2010;114(45):14605-14613.

12. Knop K, Hoogenboom R, Fischer D, Schubert US. Poly(ethylene glycol) in drug delivery: pros and cons as well as potential alternatives. Angew Chem Int Ed Engl. 2010;49(36):6288-6308.

13. Bussolino F, Marchio S, inventors; Universita Degli Studi Di Torino, assignee. Metastasis-specific peptides and their diagnostic and therapeutic applications. United States patent US 20100041614. February 18, 2012.

14. Bouvet M, Tsuji K, Yang M, Jiang P, Moossa AR, Hoffman RM. In vivo color-coded imaging of the interaction of colon cancer cells and splenocytes in the formation of liver metastases. Cancer Res. 2006;66(23):11293-11297.

15. Miyashiro I, Miyoshi N, Hiratsuka M, et al. Detection of sentinel node in gastric cancer surgery by indocyanine green fluorescence imaging: comparison with infrared imaging. Ann Surg Oncol. 2008;15(6): 1640-1643.
16. van der Poel HG, Buckle T, Brouwer OR, Valdés Olmos RA, van Leeuwen FW. Intraoperative laparoscopic fluorescence guidance to the sentinel lymph node in prostate cancer patients: clinical proof of concept of an integrated functional imaging approach using a multimodal tracer. Eur Urol. 2011;60(4):826-833.

17. D'Hallewin MA, Kamuhabwa AR, Roskams T, De Witte PA, Baert L. Hypericin-based fluorescence diagnosis of bladder carcinoma. BJU Int. 2002;89(7):760-763.

18. van Dam GM, Themelis G, Crane LM, et al. Intraoperative tumorspecific fluorescence imaging in ovarian cancer by folate receptor-alpha targeting: first in-human results. Nat Med. 2011;17(10):1315-1319.

19. de Leeuw J, van der Beek N, Neugebauer WD, Bjerring P, Neumann HA. Fluorescence detection and diagnosis of non-melanoma skin cancer at an early stage. Lasers Surg Med. 2009;41(2):96-103.

20. Atlamazoglou V, Yova D, Kavantzas N, Loukas S. Microscopical examination of the localisation patterns of two novel rhodamine derivatives in normal and neoplastic colonic mucosa. Lasers Med Sci. 2001;16(4):253-259. 


\section{Supplementary materials}

Physical characterization

Rhod tertiary amide

${ }^{1} \mathrm{H} \mathrm{NMR}\left(250 \mathrm{MHz},\left[\mathrm{D}_{3}\right] \mathrm{MeOH}, 25^{\circ} \mathrm{C}, \mathrm{TMS}\right): \delta 1.32(\mathrm{t}, 12 \mathrm{H}$, $J=7.3$ ), 3.14 (br s, 4H), 3.66-3.72 (m, 12H), 6.97-6.98 (d, $2 \mathrm{H}, J=2.2), 7.08-7.13(\mathrm{dd}, 2 \mathrm{H}, J=2.1), 7.25-7.29(\mathrm{~d}, 2 \mathrm{H}$, $J=9.8), 7.50-7.54(\mathrm{~m}, 1 \mathrm{H}), 7.77-7.82(\mathrm{~m}, 3 \mathrm{H}) ;{ }^{13} \mathrm{C}$ NMR $\left(62.9 \mathrm{MHz},\left[\mathrm{D}_{3}\right] \mathrm{MeOH}, 25^{\circ} \mathrm{C}, \mathrm{TMS}\right): \delta 13.07,39.45,44.21$, 47.06, 97.50, 114.66, 115.64, 128.96, 131.42, 131.62, 131.87, $132.32,132.89,135.53,156.57,156.98,158.96,169.25$; ESI-MS, m/z (M+H) 511.4 .

\section{Alkoxysilane-derivatized Rhod}

${ }^{1} \mathrm{H}$ NMR $\left(250 \mathrm{MHz},\left[\mathrm{D}_{3}\right] \mathrm{MeOH}, 25^{\circ} \mathrm{C}, \mathrm{TMS}\right): \delta 0.59$ (t, $2 \mathrm{H}$, $J=8.1 \mathrm{~Hz}), 1.19(\mathrm{t}, J=7.3 \mathrm{~Hz})$ and $1.33(\mathrm{t}, J=8.4 \mathrm{~Hz}) 21 \mathrm{H}$ partially overlapped, $1.57(\mathrm{~m}, 2 \mathrm{H}), 3.09(\mathrm{t}, J=6.9 \mathrm{~Hz})$ and $3.25(\mathrm{q}, J=7.3 \mathrm{~Hz}$ ) partially overlapped $8 \mathrm{H}, 3.40(\mathrm{br} \mathrm{s}, 2 \mathrm{H})$, $3.65-3.73(\mathrm{q}, J=7.3 \mathrm{~Hz})$ and $3.76-3.86(\mathrm{q}, J=7.3 \mathrm{~Hz}$, ) partially overlapped $14 \mathrm{H}, 6.97(\mathrm{~d}, J=2.6 \mathrm{~Hz}), 7.05-7.09$ (dd, $2 \mathrm{H}, J=2.6 \mathrm{~Hz}), 7.27-7.30(\mathrm{~d}, 2 \mathrm{H}, J=9.5 \mathrm{~Hz}), 7.50-7.54$ $(\mathrm{m}, 1 \mathrm{H}), 7.67-7.79(\mathrm{~m}, 3 \mathrm{H}) ;{ }^{13} \mathrm{C}$ NMR $\left(62.9 \mathrm{MHz},\left[\mathrm{D}_{3}\right]\right.$ $\mathrm{MeOH}, 25^{\circ} \mathrm{C}$, TMS): $\delta 8.52,9.10,12.88,18.70,24.60$, 44.54, 46.92, 47.54, 59.46, 97.34, 114.86, 115.46, 128.98, 131.28, 131.77, 132.32, 133.24, 136.62, 139.09, 157.12, 157.19, 159.27, 159.80, 169.50; ESI-MS, m/z (M+H) 758.6 .

Dicarboxylic acid derivative of PF-I27

${ }^{1} \mathrm{H}$ NMR (300 MHz, $\mathrm{CDCl}_{3}, 25^{\circ} \mathrm{C}, \delta \mathrm{ppm}$ ): 4.05 (s, $\left.4 \mathrm{H},-\mathrm{CH}_{2} \mathrm{COOH}\right) ; 3.52\left(\mathrm{~s},-\mathrm{OCH}_{2} \mathrm{CH}_{2} \mathrm{O}-\right), 3.43(\mathrm{~m}$, $\left.-\mathrm{OCH}_{2} \mathrm{C}-\mathrm{CH}_{3} \mathrm{O}-\right), 3.29\left(\mathrm{~m},-\mathrm{OCH}_{2} \mathrm{CHCH}_{3} \mathrm{O}-\right) 1000 \mathrm{H},(\mathrm{d}$, $\left.-\mathrm{OCH}_{2} \mathrm{CHCH}_{3} \mathrm{O}-\right) 190 \mathrm{H} ;{ }^{13} \mathrm{C}$ NMR $\left(75.7 \mathrm{MHz}, \mathrm{CDCl}_{3}\right.$, $25^{\circ} \mathrm{C}, \delta$ ppm): 75.2, 75.0, 74.8, 73.0, 72.6, 70.2, 17.1; IR ( $\mathrm{NaCl}$, thin solid film): $-\mathrm{COOH} 1735 \mathrm{~cm}^{-1}$.

Table SI Morphological and photophysical properties of the SNPs and of their doping dyes

\begin{tabular}{|c|c|c|c|c|c|c|}
\hline & \multirow[t]{2}{*}{ Solvent } & \multirow[t]{2}{*}{ Dyes/SPN } & \multicolumn{2}{|c|}{ Absorption } & \multicolumn{2}{|l|}{ Emission } \\
\hline & & & $\lambda_{\max }[\mathrm{nm}]$ & $\varepsilon\left[\mathrm{M}^{-1} \mathrm{~cm}^{-1}\right]$ & $\lambda_{\max }[\mathrm{nm}]$ & $\Phi$ \\
\hline Rhod & $\mathrm{EtOH}$ & - & 562 & $1.0 \times 10^{5}$ & 580 & 0.35 \\
\hline (Rhod)-SPNs & $\mathrm{H}_{2} \mathrm{O}$ & 4.5 & 570 & $4.5 \times 10^{5}$ & 590 & 0.45 \\
\hline Сy 5 & $\mathrm{EtOH}$ & - & 645 & $2.5 \times 10^{5}$ & 655 & 0.32 \\
\hline (Cy5)-SPNs & $\mathrm{H}_{2} \mathrm{O}$ & 3.0 & 650 & $7.5 \times 10^{5}$ & 665 & 0.34 \\
\hline \multirow[t]{2}{*}{ (Rhod+Cy5)-SNPs } & $\mathrm{H}_{2} \mathrm{O}$ & 4.0 (Rhod) & 570 & $4.0 \times 10^{5}$ & 590 & 0.04 \\
\hline & & $3.0($ (Су5) & 650 & $7.5 \times 10^{5}$ & 665 & 0.34 \\
\hline
\end{tabular}




\section{A}

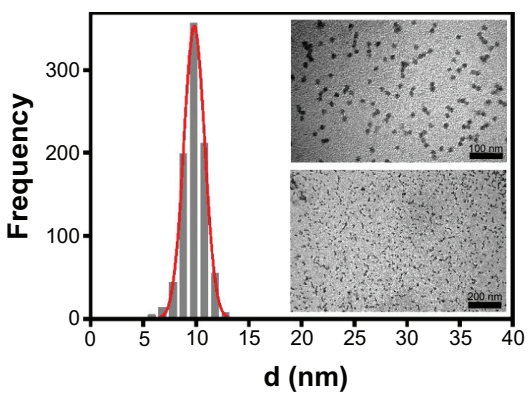

B

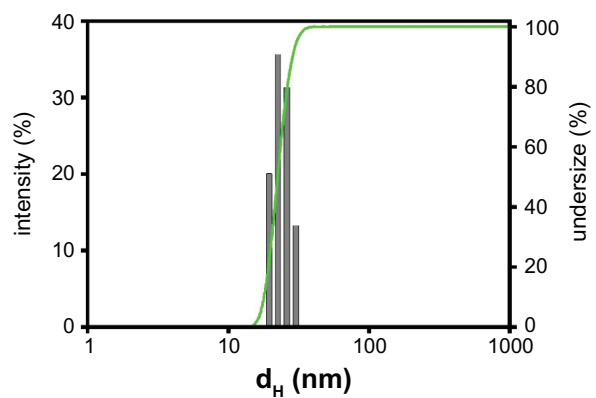

Figure SI Characterization of the fluorescent SPNs. (A) TEM analysis of SPN silica cores for the evaluation of size distribution. (B) Dynamic light scattering distribution of the nanoparticle hydrodynamic diameter. Exemplary graphs referred to CGIYRLRS-(Rhod)-SPNs are shown.

Abbreviations: SPN, silica-poly(ethylene glycol) nanoparticles; TEM, transmission electron microscopy.
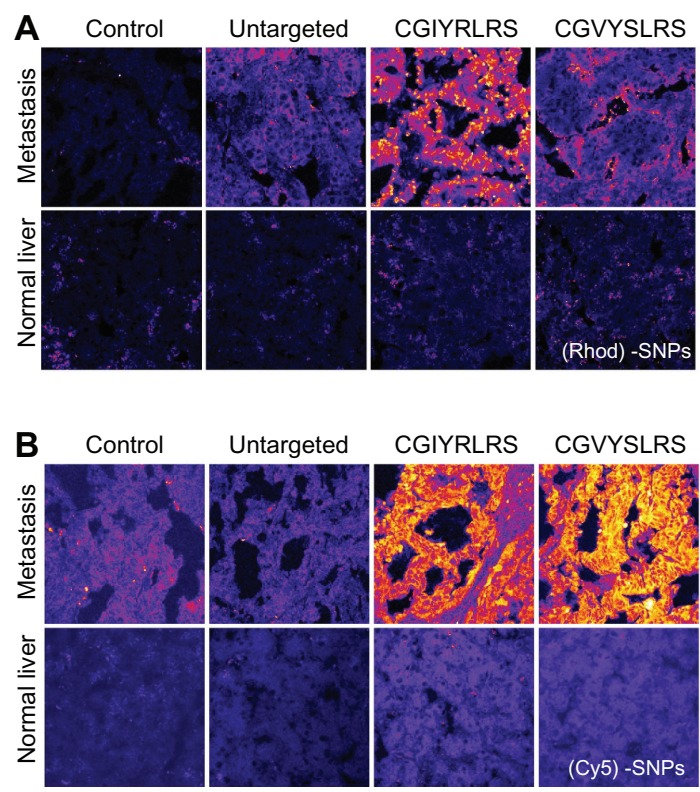

Figure S2 Single-color targeted SPNs recognize human hepatic metastases ex vivo Frozen sections (10- $\mu \mathrm{m}$ ) of matched (grossly) normal liver and hepatic metastasis were fixed in $4 \%$ formaldehyde before being incubated with $5 \times 10^{12}$ of either untargeted, CGIYRLRS-, or (A) CGVYSLRS-(Rhod)- or (B) (Cy5)-SPNs for $4 \mathrm{~h}$ at RT. After 3 washes in TBS-T, the SPN-emitted fluorescence was analyzed by confocal microscopy, and the output was converted in a false color LUT Fire scale for prompt visualization. The experiment was performed on specimens from 10 patients with metastatic CRC; exemplary images from tissues of patient \#P85 are shown.

Abbreviations: CRC, colorectal cancer; RT, room temperature; SPN, silica-poly(ethylene glycol) nanoparticles; TBS-T, Tris-buffered saline containing Tween-20.

International Journal of Nanomedicine

\section{Publish your work in this journal}

The International Journal of Nanomedicine is an international, peerreviewed journal focusing on the application of nanotechnology in diagnostics, therapeutics, and drug delivery systems throughou the biomedical field. This journal is indexed on PubMed Central,

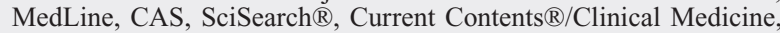

Journal Citation Reports/Science Edition, EMBase, Scopus and the Elsevier Bibliographic databases. The manuscript management system is completely online and includes a very quick and fair peer-review system, which is all easy to use. Visit http://www.dovepress.com/ testimonials.php to read real quotes from published authors. 